\title{
COMPARISON OF TWO NOVEL LIST SPHERE DETECTOR ALGORITHMS FOR MIMO-OFDM SYSTEMS
}

\author{
Markus Myllylä*, Pirkka Silvola and Markku Juntti \\ Centre for Wireless Communications \\ P.O. Box 4500, FIN-90014 University of Oulu, Finland \\ \{markus.myllyla, pirkka.silvola, markku.juntti\}@ee.oulu.fi
}

\author{
Joseph R. Cavallaro \\ Dept. of Electrical \& Computer Engineering \\ Rice University, Houston, TX 77251-1892, USA \\ cavallar@rice.edu
}

\section{ABSTRACT}

In this paper, the complexity and performance of two novel list sphere detector (LSD) algorithms are studied and evaluated in multiple-input multiple-output orthogonal frequency division multiplexing (MIMO-OFDM) system. The LSDs are based on the K-best and the Schnorr-Euchner enumeration (SEE) algorithms. The required list sizes for LSD algorithms are determined for a $2 \times 2$ system with 4 - quadrature amplitude modulation (QAM), 16-QAM, and 64-QAM. The complexity of the algorithms is compared by studying the number of visited nodes per received symbol vector by the algorithm in computer simulations. The SEE based LSD algorithm is found to be a less complex and a feasible choice for implementation compared to the K-best based LSD algorithm.

\section{INTRODUCTION}

The increasing data rates in wireless communication systems require large bandwidths. Orthogonal frequency division multiplexing (OFDM) [1] has become a widely used technique to significantly reduce receiver complexity in broadband wireless systems. Multiple-input multiple-output (MIMO) channels offer improved capacity and significant potential for improved reliability compared to single antenna channels [2]. MIMO techniques in combination with OFDM (MIMO-OFDM) have been identified as a promising approach for high spectral efficiency wideband systems [3].

The OFDM technique drastically simplifies receiver design by decoupling the intersymbol interference, i.e., a frequency selective, MIMO channel into a set of parallel flat fading MIMO channels [3]. However, the reception of the MIMOOFDM signal has to be performed separately for each subcarrier. The optimal detection for coded system would require the use of a maximum a posteriori (MAP) detection. However, the computational complexity of optimal MAP detection is beyond the limit of most systems, and, thus, such an approach is not feasible. A suboptimal approach is to use suboptimal zero forcing $(\mathrm{ZF})$ or minimum mean square error (MMSE) criterion based linear detectors [4]. However, their performance can be rather poor in bad channel conditions, i.e., when the eigenvalue spread of the channel matrix is large [5].

Sphere detector (SD) calculates the maximum likelihood (ML) solution with reduced complexity compared to fullcomplexity ML detectors $[6,7,8]$. A list sphere detector (LSD)

\footnotetext{
${ }^{*}$ This work was done in MITSE project which was supported by Elektrobit, Nokia, Texas Instruments and the Finnish Funding Agency for Technology and Innovation, Tekes. The first author would also like to thank Nokia foundation for supporting the research.
}

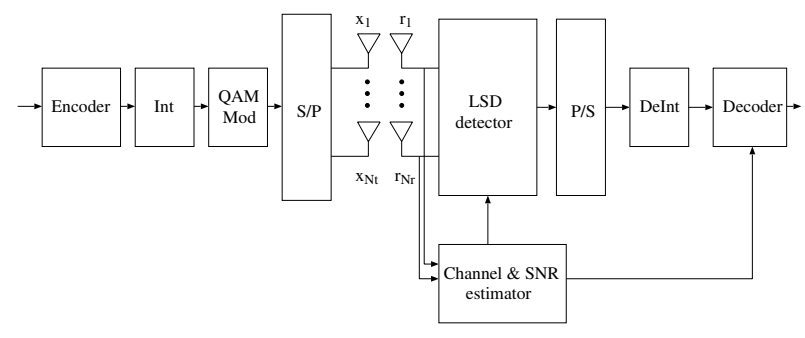

Figure 1: Model of a MIMO system with $N_{T}$ transmit and $N_{R}$ receive antennas.

[9] is a variant of the sphere detector that can be used to approximate MAP detection. In this paper, two novel LSD algorithms are introduced: a modification of the Schnorr-Euchner enumeration (SEE) [10] to the LSD algorithm, namely a SEELSD algorithm, and a modification of the K-best algorithm [11] to LSD algorithm, namely a K-best-LSD algorithm. The complexity and the performance of the algorithms are evaluated and compared.

The paper is organized as follows. The system model is presented in Section II. The LSD algorithms are introduced in Section III. Performance examples are presented in Section IV. Conclusions are drawn in Section V.

\section{SYSTEM MODEL}

An OFDM based multiple antenna system with $N_{T}$ transmit antennas and $N_{R}$ receive antennas is considered with assumption $N_{R} \geq N_{T}$. A vertical Bell Labs' layered space-time (VBLAST) architecture [12] with quadrature amplitude modulation (QAM) is applied to the system. A block diagram of the system is shown in Figure 1. The received signal can be expressed in terms of code symbol interval as

$$
\mathbf{r}_{p}=\mathbf{H}_{p} \mathbf{x}_{p}+\boldsymbol{\eta}_{p}, \quad p=1,2, \ldots, P,
$$

where $P$ is the number of subcarriers, the received signal vector $\mathbf{r}_{p} \in \mathbb{C}^{N_{R} \times 1}$, the transmit symbol vector $\mathbf{x}_{p} \in \mathbb{C}^{N_{T} \times 1}$ and the noise vector $\boldsymbol{\eta}_{p} \in \mathbb{C}^{N_{R} \times 1}$ are defined in the frequency domain. The elements of $\boldsymbol{\eta}_{p}$ are independent and complex Gaussian with equal power real and imaginary parts, i.e., $\boldsymbol{\eta}_{p} \sim \mathcal{C N}\left(\mathbf{0}, N_{0} \mathbf{I}_{N_{R}}\right)$ and represent the frequency domain thermal noise at the receiver. The channel matrix $\mathbf{H}_{p} \in \mathbb{C}^{N_{R} \times N_{T}}$ contains complex Gaussian fading coefficients with unit variance. The entries of $\mathbf{x}_{p}$ are chosen independently from a complex QAM constellation $\Omega$ with $Q$ bits per symbol, i.e., $|\Omega|=2^{Q}$. The set of all possible transmitted vector symbols 
is denoted by $\Omega^{N_{T}}$. The corresponding uncoded transmission rate is $R=M_{T} Q$ bits per channel use (bpcu).

ML detection method solves optimally so called closest lattice point problem by calculating the Euclidean distances (EDs) between received signal $\mathbf{y}$ and lattice points $\mathbf{H x}$ and decides which lattice point minimizes the Euclidean distance to receive vector $\mathbf{y}$, i.e.,

$$
\hat{\mathbf{x}}_{\mathrm{ML}}=\arg \min _{\mathbf{x} \in \Omega^{N_{T}}}\|\mathbf{y}-\mathbf{H x}\|_{2}^{2} .
$$

For simplicity, the subindices are omitted in (2) and in the sequel. The ML detection problem can be solved with an exhaustive search, i.e., checking all of the possible symbol vectors and selecting the closest point. However, this is computationally very heavy and not feasible as the set of possible points $\Omega^{N_{T}}$ increases.

The SD algorithms solve the ML solution by reducing the number of candidate vector symbols to be considered in the search that solves (2). Usually SD algorithms are assumed with a real equivalent system model which makes the search process easier to implement. The complex MIMO system model in (1) can be reduced into equivalent real model as follows

$$
\left[\begin{array}{l}
\operatorname{Re}(\mathbf{r}) \\
\operatorname{Im}(\mathbf{r})
\end{array}\right]=\left[\begin{array}{cc}
\operatorname{Re}(\mathbf{H}) & -\operatorname{Im}(\mathbf{H}) \\
\operatorname{Im}(\mathbf{H}) & \operatorname{Re}(\mathbf{H})
\end{array}\right]\left[\begin{array}{l}
\operatorname{Re}(\mathbf{x}) \\
\operatorname{Im}(\mathbf{x})
\end{array}\right]+\left[\begin{array}{l}
\operatorname{Re}(\eta) \\
\operatorname{Im}(\eta)
\end{array}\right] .
$$

Let us define the new real dimensions $M_{\mathrm{T}}=2 N_{\mathrm{T}}, M_{\mathrm{R}}=$ $2 N_{\mathrm{R}}$. The real symbol alphabet is now $\Omega_{R}=\mathbb{Z}$, e.g., $\Omega_{R} \in$ $\{-3,-1,1,3\}$ in the case of 16-QAM. The SD algorithm reduces the number of considered candidate symbol vectors by limiting the search to points that lie inside a $M_{\mathrm{R}}$-dimensional hyper-sphere $S\left(\mathbf{y}, \sqrt{C_{0}}\right)$ centered at $\mathbf{y}$. The condition can be written as [7]

$$
\|\mathbf{y}-\mathbf{H x}\|_{2}^{2} \leq C_{0},
$$

where $C_{0}$ is the squared radius of the the sphere. The channel matrix $\mathbf{H}$ can be decomposed by QR decomposition (QRD) and then (4) can be written as

$$
\begin{aligned}
\|\mathbf{y}-\mathbf{Q R \mathbf { x }}\|_{2}^{2} & \leq C_{0} \\
\left\|\mathbf{Q}^{\mathrm{T}} \mathbf{y}-\mathbf{R x}\right\|_{2}^{2} & \leq C_{0} \\
\|\tilde{\mathbf{y}}-\mathbf{R x}\|_{2}^{2} & \leq C_{0},
\end{aligned}
$$

where $\mathbf{R} \in \mathbb{R}^{M_{T} \times M_{T}}$ is an upper triangular matrix with positive diagonal elements, $\mathbf{Q} \in \mathbb{R}^{M_{R} \times M_{T}}$ is a orthogonal matrix, and $\tilde{\mathbf{y}}=\mathbf{Q}^{\mathrm{T}} \mathbf{y}$.

Let $\mathbf{x}_{i}^{M_{\mathrm{T}}}=\left(x_{i}, x_{i+1}, \ldots, x_{M_{\mathrm{T}}}\right)^{\mathrm{T}}$ denote the last $M_{\mathrm{T}}-i+1$ components of the vector $\mathbf{x}$. The sphere search can be illustrated with a tree structure, where the root layer corresponds to $\mathbf{x}_{M_{T}}^{M_{\mathrm{T}}}$. A sphere search tree structure is illustrated in Figure 2. Due to the upper triangular form of $\mathbf{R}$ the values of $\mathbf{x}$ can be solved from (7) level by level using the back-substitution algorithm. First, the last elements of the possible symbol vectors are calculated, i.e, $x_{M_{\mathrm{T}}}$ and then $x_{M_{\mathrm{T}-1}}$ and so on. The squared partial ED (PED) of $\mathbf{x}_{i}^{M_{T}}$ can be calculated as

$$
d\left(\mathbf{x}_{i}^{M_{T}}\right)=\sum_{j=i}^{M_{T}}\left|\tilde{y}_{j}-\sum_{l=j}^{M_{T}} r_{j, l} x_{l}\right|^{2} \leq C_{0}
$$

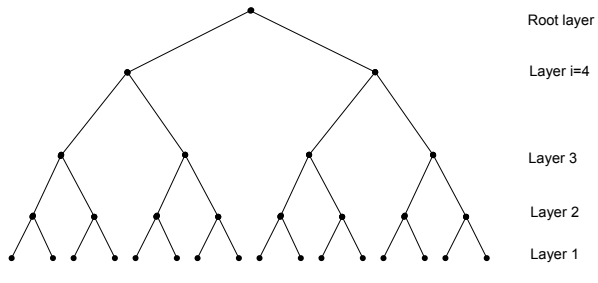

Figure 2: A tree structure of a sphere detector with 2 transmit, 2 receive antennas and 4-QAM modulation

where $r_{j, l}$ is the $(j, l)$ th term of $\mathbf{R}$ and $i=M_{\mathrm{T}}, \ldots, 1$. At each level the next admissible nodes are checked by calculating the PEDs or with real presentation with equations presented, e.g., in [7]. The search for the particular path is stopped if the PED of a node $\mathbf{x}_{i}^{M_{\mathrm{T}}}$ is outside the sphere, i.e., $d\left(\mathbf{x}_{i}^{M_{T}}\right)>C_{0}$. The SD searches the lattice points inside the sphere $\mathbf{H x} \in S\left(\mathbf{y}, \sqrt{C_{0}}\right)$, and selects the point $\mathbf{x}=\mathbf{x}_{1}^{M_{T}}$, also called as a leaf node, for which the $\operatorname{ED} d\left(\mathbf{x}_{1}^{M_{T}}\right)$ is minimum. It should be noted that with too small a sphere radius $\sqrt{C_{0}}$ there might be no solutions [7].

The Pohst enumeration method [6] is often considered to be the original sphere detection algorithm. However, the main problem with the algorithm is the selection of the sphere radius. The Viterbo-Boutros (VB) implementation [13] introduced a modification to the original Pohst algorithm with adaptive updating of the sphere radius as a new solution $\mathbf{x}_{1}^{M_{T}}$ is found. Thus, with VB implementation applied, the algorithm can be started with $C_{0}=\infty$. The Schnorr Euchner enumeration (SEE) [10] can be seen as a modification of the Pohst enumeration and VB implementation, where the admissible nodes of each layer are spanned in a zig-zag order starting with the closest middle point, whereas the Pohst enumeration searches the admissible nodes without any ordering. The SEE has been shown to be more efficient than the VB implementation [8].

The SD algorithms give the ML solution as an output. However, the performance of a channel coded system may suffer significantly with ML detection prior soft input decoding and iterative processing at the receiver compared to the optimal MAP detection. The list sphere detector (LSD) [9] can be used for obtaining a list of the most probable candidate symbol vectors $\mathcal{L} \in \mathbb{Z}^{N_{\text {cand }} \times N_{\mathrm{T}}}$ as an output, where $N_{\text {cand }}$ is the size of the candidate list so that $1 \leq N_{\text {cand }} \leq 2^{Q N_{\mathrm{T}}}$. The list can then be used to approximate the MAP solution. Depending on the list size $N_{\text {cand }}$, it provides a tradeoff between the performance and the computational complexity.

\section{List SPHERE Detector}

A basic block diagram of the list sphere detector structure is shown in Figure 3. The LSD gives log-likelihood ratios (LLR) of the transmitted bits as an output. The LSD structure consists of four parts: the QRD block, the LSD algorithm block, the demodulator block and the LLR calculation block.

A standard preprocessing needed for sphere detection is the QRD of the channel matrix $\mathbf{H}[7,14]$. The preprocessing should be repeated for each subcarrier $p$ every time when the channel realization changes, i.e., it depends on the channel coherence time. The complexity of the QRD block is mainly de- 


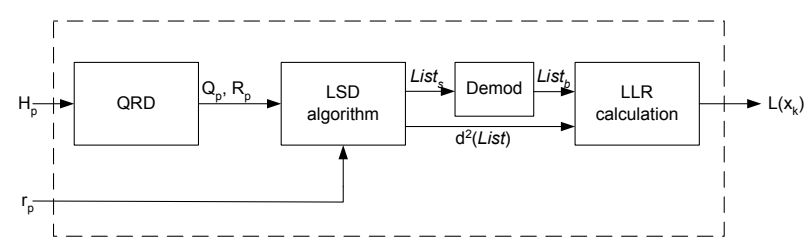

Figure 3: A block diagram of a list sphere detector structure.

pendent on the matrix dimensions of the channel matrix $\mathbf{H}$ and the number of subcarriers $P$. The QRD implementation is discussed, e.g., in [15, 16, and referencens therein].

The LSD gives a list of the most probable transmitted symbols $\mathcal{L}$ and the corresponding Euclidean distances $d(\mathcal{L})$ as an output. The LSD algorithm operates at symbol rate and it should calculate the outputs separately for each subcarrier. The complexity of the LSD depends on the applied algorithm, the list size $N_{\text {cand }}$, the number of subcarriers $P$, the number of transmit antennas $N_{T}$ and the constellation size $|\Omega|$. These variables affect to the expected number of visited nodes per received symbol vector. In the algorithm itself, the main complexity comes from the calculation of PEDs [14]. It should be noted that different lattice reduction preprocessing [7, 14] approaches may yield a lower expected number of visited nodes per symbol and, thus, lower complexity.

Two novel LSD algorithms are considered in this paper. The SEE-LSD algorithm presented as Algorithm 1 is a modification of the SEE [10] to LSD algorithm and the K-best-LSD algorithm presented as Algorithm 2 is a modification of the K-best algorithm $[11,17]$ to LSD algorithm.

\section{Algorithm 1 (SEE-LSD algorithm)}

\section{Preprocessing:}

Input: $\mathbf{Q}, \mathbf{R}, \mathbf{y}, N_{\text {cand }}, P$ (modulation used, $\left.P-Q A M\right)$

Calculate: $\tilde{\mathbf{y}}$, Set: $C_{0}=\infty, k=1$

\section{Algorithm:}

1. Start with empty candidate set from the root layer.

2. Denote the partial candidate set by $\boldsymbol{x}_{i+1}^{M_{T}}$. If all admissible nodes at layer $i$ are searched go to step 2.1. Otherwise, generate the next best admissible node $x_{i}$ using SEE, calculate $d\left(\boldsymbol{x}_{i}^{M_{T}}\right)$ and go to step 3 .

2.1 If $i+1$ is the root layer, stop the algorithm and give the final list and the EDs as an output. Otherwise, continue to step 2 at layer $i+2$ with $\boldsymbol{x}_{i+2}^{M_{T}}$.

3. If the generated candidate is a leaf node $\boldsymbol{x}_{1}^{M_{T}}$ continue to step 3.1. Otherwise, go to step 4.

3.1 If $d\left(\boldsymbol{x}_{1}^{M_{T}}\right) \leq C_{0}$, the newly generated candidate leaf node $\boldsymbol{x}_{1}^{\bar{M}_{T}}$ is stored to the final list with index $k$.

Otherwise, discard the node, continue to step 2 with $\boldsymbol{x}_{i+2}^{M_{T}}$.

3.2 If $k=N_{\text {cand }}$, find the candidate with maximum

ED and set $C_{0}=d_{\max }$ and $k=k_{d_{\max }}$. Otherwise,

increase the final list index $k=k+1$.

3.3 Continue to step 2 with $\boldsymbol{x}_{i+1}^{M_{T}}$.

4. If $d\left(\boldsymbol{x}_{i}^{M_{T}}\right) \leq C_{0}$ go to step 2 and continue with the generated candidate child node $x_{i}^{M_{T}}$. Otherwise, discard the node and continue to step 2.1 with $\boldsymbol{x}_{i+2}^{M_{T}}$.
The SEE-LSD algorithm has only one major difference compared to the normal SEE-SD algorithm: the sphere radius $C_{0}$ is not updated until the final list is full. After that the search is continued in similar fashion until the admissible nodes have been searched.

\section{Algorithm 2 (K-best-LSD algorithm)}

Preprocessing:

Input: $\mathbf{Q}, \mathbf{R}, \mathbf{y}, C_{0}, K, P$ (modulation used, $P-Q A M$ )

Calculate: $\tilde{\mathbf{y}}$

Algorithm:

1. Start with empty candidate set from the root layer.

2. Denote the partial candidate set by $\boldsymbol{x}_{i+1}^{M_{T}}$.

2.1 Determine all admissible candidate child nodes $x_{i}$ with given $C_{0}$ and calculate the corresponding PEDs $d\left(\boldsymbol{x}_{i}^{M_{T}}\right)$.

2.2 Store the partial candidates and their PEDs to a temporary stack memory.

3. Sort the partial candidates according to their PEDs and store the $K$ candidates with lowest PEDs and the PEDs to the final list stack memory.

4. If the K stored candidates are leaf nodes, stop the algorithm and give the candidates and their EDs as outputs. Otherwise, continue to step 2 with the stored candidates.

The only major change in the LSD version of the algorithm compared to the SD algorithm with SEE [17] is that with Kbest-LSD algorithm in the end all $\mathrm{K}$ candidates are given as outputs as with K-best algorithm only the candidate with lowest ED is given as an output.

The output candidate symbol vector list from the LSD algorithm is demodulated to bit level representation in the demodulator block. Then, the LLRs of the transmitted bits can be calculated from the candidate bit vector list in the LLR calculation block. The LLR of transmitted bit $x_{k}$ is is denoted as $L\left(x_{k}\right)$ and is defined to be the ratio of the probabilities of the bit taking its two possible values, i.e.,

$$
L\left(x_{k}\right)=\ln \frac{\operatorname{Pr}\left(x_{k}=+1 \mid \mathbf{r}\right)}{\operatorname{Pr}\left(x_{k}=-1 \mid \mathbf{r}\right)} .
$$

The probability of a transmitted bit $x_{k}=+1$ is equal to the sum of the probabilities of all of the combinations containing a $x_{k}=+1$ for that given bit. Then, for a system containing additive white Gaussian noise (AWGN), the probability can be determined directly from the cost information known about the candidates and Bayes rule as

$$
p\left(\mathbf{r} \mid x_{k}=1\right)=\frac{2}{\left|\Omega_{R}\right|^{M_{T} \sqrt{2 \pi \sigma^{2}}}} \sum_{\mathbf{x} \in \Omega_{R}^{M_{T}}, x_{k}=+1} e^{\frac{-d\left(\mathbf{x}_{1} M_{T}\right)}{2 \sigma^{2}}}
$$

If the size $N_{\text {cand }}$ of the list $\mathcal{L}$ is large enough, the the effect of the unknown results is likely to be relatively small, and approximation of the $L\left(x_{k}\right)$ can then be determined as

$$
\begin{aligned}
L\left(x_{k}\right) & =\ln \frac{\operatorname{Pr}\left(x_{k}=+1 \mid \mathbf{r}\right)}{\operatorname{Pr}\left(x_{k}=-1 \mid \mathbf{r}\right)} \\
& =\ln \left(p\left(\mathbf{r} \mid x_{k}=+1\right)-\ln \left(p\left(\mathbf{r} \mid x_{k}=-1\right)\right)\right.
\end{aligned}
$$

Equation (11) can then be computed using the well-known Jacobian logarithm and a small look-up table [18]. 


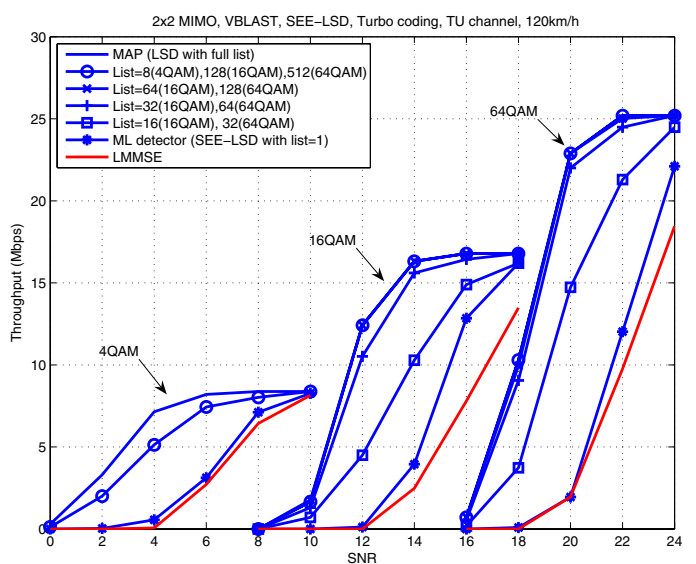

Figure 4: Throughput vs SNR: Performance of the SEE-LSD algorithm with different list sizes in $2 \times 2$ antenna system.

\begin{tabular}{c|c}
\multicolumn{2}{c}{ Table 1: The determined list sizes. } \\
\hline $2 \times 2$ with 4-QAM & $N_{\text {cand }}=16$ \\
$2 \times 2$ with 16-QAM & $N_{\text {cand }}=64$ \\
$2 \times 2$ with 64-QAM & $N_{\text {cand }}=128$
\end{tabular}

\section{PERFORMANCE EXAMPLES}

The effect of the list size to the performance of SEE-LSD and K-best-LSD was studied via computer simulations. A $2 \times 2$ MIMO-OFDM system was assumed with 512 subcarriers (300 used). A turbo coded VBLAST architecture with $1 / 2$ code rate was applied in a typical urban (TU) 6 tap channel with a user velocity of $120 \mathrm{kmph}$. The system was operating with $5 \mathrm{MHz}$ bandwidth at a carrier frequency of $2.4 \mathrm{GHz}$. The considered LSD algorithms were studied with different list sizes and compared to a ML detector, which corresponds to a SEE-LSD with list size of one, and to a linear MMSE (LMMSE) detector at the receiver side. Soft outputs of the detectors were decoded in an iterative turbo decoder with 8 iterations. The parameters used in the computer simulations correspond to the parameters proposed in [19] for initial performance evaluation in 3G LTE. The K-best-LSD algorithm was applied with $C_{0}=\infty$.

The throughput of the SEE-LSD and K-best-LSD algorithms with different list sizes and with different modulations is presented in Figures 4 and 5, respectively. The results illustrate the effect of the list size to the quality of the MAP approximation in (11). The maximum list sizes $N_{\text {cand }}^{\max }$ for 4-, 16- and 64-QAM modulations, that correspond to optimal MAP detection outputs, are 16, 256, and 4096, respectively. It can be seen from the results that the performance of the LSD algorithms is almost the same in each case and the minimum required list size without significant performance loss can be determined. The 4-QAM requires a full list of 16 candidates, and with 16-QAM a list size of 64 candidates is needed. The 64-QAM requires a list size of 128 candidates. It is noted that list sizes of 32 and 64 candidates can also be considered with slight performance loss for 16-QAM and 64-QAM, respectively. The determined list sizes $N_{\text {cand }}$ are shown in Table 1 .

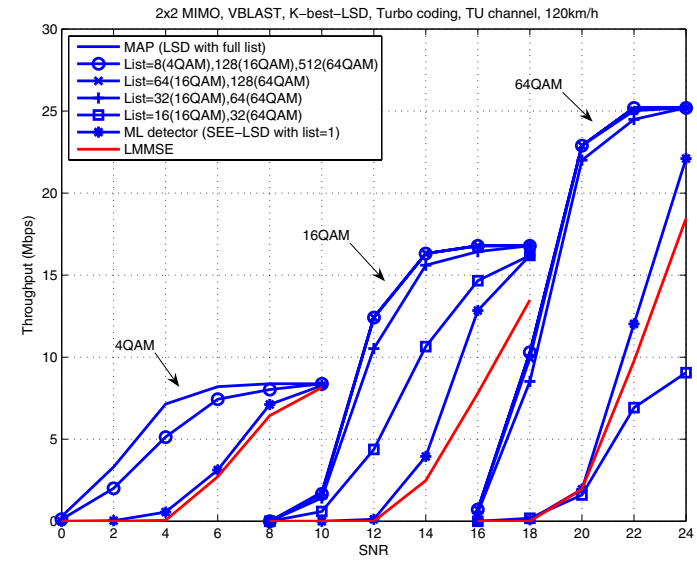

Figure 5: Throughput vs SNR: Performance of the K-best-LSD with different list sizes in $2 \times 2$ antenna system.

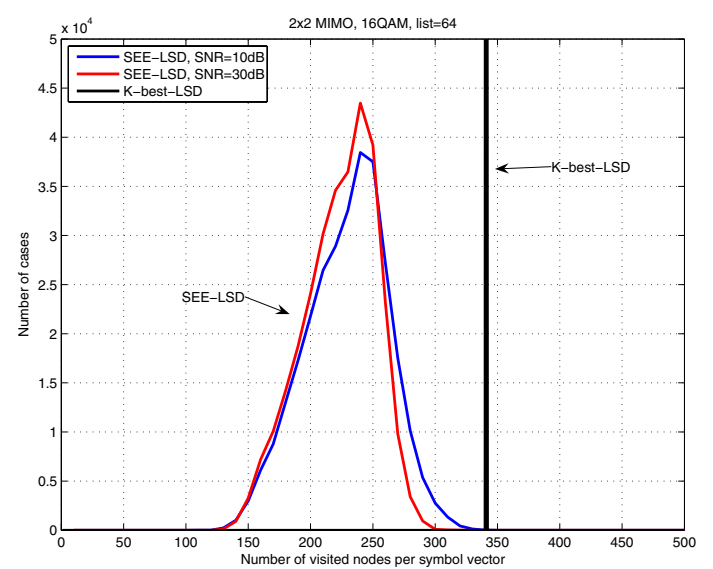

Figure 6: Histogram of the number of visited nodes per symbol vector with the SEE-LSD and the K-best-LSD algorithms in TU channel with $2 \times 216$-QAM system.

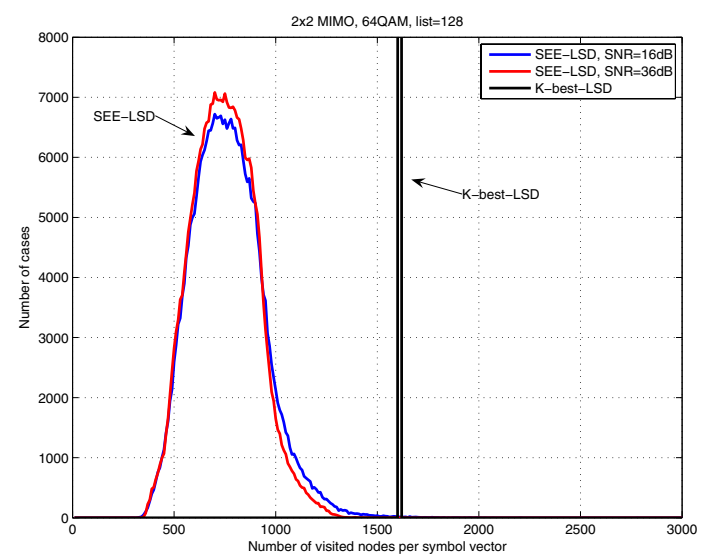

Figure 7: Histogram of the number of visited nodes per symbol vector with the SEE-LSD and the K-best-LSD algorithms in TU channel with $2 \times 264-\mathrm{QAM}$ system. 
The complexity of the LSD algorithms is relative to the number of visited nodes per symbol vector [14]. The number of nodes visited by the considered LSD algorithms was studied with determined list sizes via computer simulations. The 4QAM requires a full list size of 16 candidates, and, thus, it visits every possible node. A histogram of the simulated number of visited nodes per symbol vector with the SEE-LSD and the K-best-LSD algorithms with list size of 64 candidates with 16-QAM modulation is shown in Figure 6. The number of visited nodes with the SEE-LSD algorithm varies slightly with SNR and with the K-best-LSD algorithm it is fixed. This is due to different search strategies of the algorithms. It can be seen from Figure 6 that the SEE-LSD algorithm is likely to visit less nodes compared to K-best-LSD algorithm. However, it should be noted that the implementation is usually designed according the worst case scenario. A histogram of the simulated number of visited nodes per symbol vector with the SEE-LSD and the K-best-LSD algorithms with list size of 128 candidates with 64-QAM modulation is shown in Figure 7. It can be seen that the number of visited nodes is smaller even in the worst case with the SEE-LSD algorithm. Thus, the SEE-LSD algorithm seems to be a more attractive choice for implementation.

\section{COnclusions}

The complexity and performance of two novel LSD algorithms, namely the SEE-LSD and the K-best-LSD, have been evaluated and compared in MIMO-OFDM system. The needed list sizes are determined for $2 \times 2$ antenna system with 4-,16, and 64QAM modulations. Also the average number of nodes visited by the algorithms was studied with the determined list sizes. The SEE-LSD algorithm was found to be less complex and feasible choice for implementation compared to the K-best based LSD algorithm.

\section{ACKNOWLEDGEMENTS}

The authors gratefully acknowledge MITSE project team and sponsors for their suggestions and comments.

\section{REFERENCES}

[1] L. J. Cimini, "Analysis and simulation of a digital mobile channel using orthogonal frequency division multiplexing," IEEE Trans. Commun., vol. 33, no. 7, pp. 665-675, July, 1985.

[2] A. Goldsmith, S.A. Jafar, N. Jindal, and S. Vishwanath, "Capacity limits of MIMO channels," IEEE Journal on Selected Areas in Communications, vol. 21, no. 5, pp. 684-702, June, 2003.

[3] H. Yang, "A road to future broadband wireless access: MIMOOFDM-based air interface," Communications Magazine, IEEE, vol. 43, no. 1, pp. 53-60, Jan., 2005.

[4] G.J. Foschini, "Layered space-time architecture for wireless communication in a fading environment when using multielement antennas," vol. 1, no. 2, pp. 41-59, Aug. 1996.

[5] D. Wübben, R. Böhnke, V. Kühn, and K. Kammeyer, "Nearmaximum-likelihood detection of MIMO systems using MMSEbased lattice-reduction," in Proc. IEEE Int. Conf. Commun. (ICC), Paris, France, June 20-24 2004, vol. 2, pp. 798-802.
[6] U. Fincke and M. Pohst, "Improved methods for calculating vectors of short length in a lattice, including a complexity analysis," Math. Comput., vol. 44, no. 5, pp. 463-471, May 1985.

[7] M. O. Damen, H. El Gamal, and G. Caire, "On maximumlikelihood detection and the search for the closest lattice point," IEEE Trans. Inform. Theory, vol. 49, no. 10, pp. 2389-2402, Oct. 2003.

[8] E. Agrell, T. Eriksson, A. Vardy, and K. Zeger, "Closest point search in lattices," IEEE Trans. Inform. Theory, vol. 48, no. 8, pp. 2201-2214, Aug. 2002.

[9] B. Hochwald and S. ten Brink, "Achieving near-capacity on a multiple-antenna channel," IEEE Trans. Commun., vol. 51, no. 3, Mar. 2003.

[10] C. P. Schnorr and M. Euchner, "Lattice basis reduction: Improved practical algorithms and solving subset sum problems," Math. Programming, vol. 66, no. 2, pp. 181-191, Sept. 1994.

[11] K.Wong, C. Tsui, R.-K. Cheng, and W. Mow, "A VLSI architecture of a K-best lattice decoding algorithm for MIMO channels," in Proc. IEEE ISCAS'02, Helsinki, Finland, June 11-14 2002, vol. 3, pp. 273-276.

[12] P. W. Wolniansky, G. J. Foschini, G. D. Golden, and R. A. Valenzuela, "V-BLAST: An architecture for realizing very high data rates over the rich-scattering wireless channel," in International Symposium on Signals, Systems, and Electronics (ISSSE), Pisa, Italy, Sept. 29-Oct. 2 1998, pp. 295-300.

[13] E. Viterbo and J. Boutros, "A universal lattice code decoder for fading channels," IEEE Trans. Inform. Theory, vol. 45, no. 5, pp. 1639-1642, July 1999.

[14] Andreas Burg, Moritz Borgmann, Markus Wenk, Martin Zellweger, Wolfgang Fichtner, and Helmut Bölcskei, "VLSI Implementation of MIMO Detection Using the Sphere Decoding Algorithm," IEEE Journal of Solid-State Circuits, vol. 40, no. 7 , July 2005.

[15] M. Myllylä, M. Vehkaperä, and M. Juntti, "Complexity Evaluation of MMSE Based Detector for LST Architectures," in In Proc. IEEE International Workshop on Convergent Technologies (IWCT'05), Oulu, Finland, 6-10 June, 2005.

[16] M. Myllylä, J-M Hintikka, M. Limingoja, A. Byman, J.R. Cavallaro, and M. Juntti, "Complexity Analysis of MMSE Detector Architectures for MIMO OFDM Systems," in Asilomar Conference on Signals, Systems and Computers, Monterey, USA, Oct 10 - Nov 2, 2005.

[17] Zhan Guo and Peter Nilsson, "VLSI Implementation Issues of Lattice Decoders for MIMO Systems," in Circuits and Systems, ISCAS '04, 2004, vol. 4, pp. IV - 477-80.

[18] P. Robertson, E. Villebrun, and P. Hoeher, "A comparison of optimal and sub-optimal MAP decoding algorithms operating in the log domain," Proc. IEEE Int. Conf. Commun. (ICC), pp. 1009-1013, 1995.

[19] 3rd Generation Partnership Project (3GPP), "TSGR1\#41 R1050-520, EUTRA downlink numerology,” Tech. Rep., 3rd Generation Partnership Project (3GPP), 2005. 